\title{
Bibliothèques de classe de maternelle 4 ans : quelle offre pour les enfants en milieu défavorisé ?
}

Elaine Turgeon, Annie Charron et Sarah Jane McKinley

\section{OpenEdition}

Journals

Édition électronique

URL : https://journals.openedition.org/trema/6594

DOI : $10.4000 /$ trema.6594

ISSN : 2107-0997

Éditeur

Faculté d'Éducation de l'université de Montpellier

Référence électronique

Elaine Turgeon, Annie Charron et Sarah Jane McKinley, «Bibliothèques de classe de maternelle 4 ans : quelle offre pour les enfants en milieu défavorisé ? », Tréma [En ligne], 55 | 2021, mis en ligne le 20 octobre 2021, consulté le 13 décembre 2021. URL : http://journals.openedition.org/trema/6594 ; DOI : https://doi.org/10.4000/trema.6594

Ce document a été généré automatiquement le 13 décembre 2021.

Trema 


\title{
Bibliothèques de classe de maternelle 4 ans : quelle offre pour les enfants en milieu défavorisé ?
}

\author{
Elaine Turgeon, Annie Charron et Sarah Jane McKinley
}

\section{Introduction}

1 Plusieurs études ont démontré qu'un environnement physique de qualité soutient le développement des habiletés en émergence de l'écrit ${ }^{1}$ des enfants à l'éducation préscolaire $^{2}$ (Guo et al., 2012 ; Morrow et al., 2019 ; Tracey et Morrow, 2015). Les enfants issus de milieux défavorisés et ceux dont le français n'est pas la langue maternelle en bénéficieraient particulièrement (Barnett et al., 2007 ; Barnett et al., 2005 ; Gormley et Gayer, 2005). Un tel environnement comprend notamment un coin-lecture et une bibliothèque de classe bien aménagée, accessible et dotée d'une offre de lecture riche et variée (Giasson, 2011 ; Neuman et Bredekamp, 2000 ; Neuman et Roskos, 2007 ; Tracey et Morrow, 2015 ; Vukelich et al., 2012). Le terme coin-lecture fait référence à l'espace physique où les enfants peuvent s'installer pour lire tandis que le terme bibliothèque de classe désigne le mobilier où les livres sont rangés afin d'être accessibles aux enfants (Frier et Vadcar, 2017).

2 La mission de la maternelle 4 ans à temps plein en milieu défavorisé (TPMD) ${ }^{3}$ est d'améliorer les chances de réussite des enfants issus de milieux défavorisés. Pour y parvenir, il importe que la qualité de l'environnement oral et écrit des classes qui accueillent ces enfants soit élevée (Marjanovic Umek et al., 2007). En particulier, les bibliothèques de classe doivent présenter des caractéristiques qui leur permettent de contribuer à combler l'écart qui existe entre les milieux socio-économiquement contrastés (Neuman et Celano, 2001). Or, les écoles en milieu défavorisé offrent souvent des environnements de moindre qualité comparativement à celles situées en milieu favorisé, ce qui contribue davantage à accentuer l'écart qu'à le compenser en ce qui concerne les ressources dont les enfants disposent à la maison (Salinas, 2018). 
Il existe peu d'outils pour évaluer la qualité de l'environnement oral et écrit des classes de maternelle et ceux qui existent, par exemple le Early Language and Literacy Classroom Observation Pre-K [ELLCO] de Smith et ses collaborateurs (2008), s'intéressent de façon globale à la qualité de l'environnement physique et ne permettent pas d'étudier de façon détaillée le contenu des bibliothèques de classe. De plus, il y a peu d'études qui portent spécifiquement sur la qualité des bibliothèques de classe, et plus particulièrement en contexte de classe maternelle québécoise située en milieu défavorisé. Il nous semble nécessaire de nous y intéresser étant donné que les enfants de ces milieux ont habituellement accès, dans leur famille, à moins de livres et que ces derniers sont plus souvent de type commercial, puisque moins onéreux, comme l'a montré Bonnéry (2014) dans une étude menée en France auprès de familles au profil socioéconomique contrastré. En outre, dans les quartiers défavorisés, les endroits pour se procurer des livres sont moins nombreux ou moins spécialisés (ce sont par exemple des magasins d'aubaines plutôt que des librairies) (Neuman et Celano, 2001). Ajoutons qu'aucune liste ni aucun choix de corpus ne sont actuellement prescrits au Québec, contrairement à la France ${ }^{4}$, ce qui peut laisser les enseignants, qui sont peu ou pas formés à la littérature jeunesse ${ }^{5}$, plutôt démunis quant aux meilleurs choix à effectuer (Turgeon, 2013).

4 L'intention de cet article est de présenter des résultats qui s'inscrivent dans une plus vaste étude menée au Québec dans 30 classes de maternelle 4 ans à temps plein en milieu défavorisé (TPMD) ${ }^{6}$, dont l'objectif était notamment d'évaluer la qualité de l'environnement oral et écrit. De façon plus spécifique, nous présentons les résultats d'un volet de l'étude qui visait à décrire les bibliothèques de classe et à en évaluer la qualité .

\section{Cadre théorique}

Divers critères sont utilisés par les chercheurs pour évaluer le niveau de qualité des bibliothèques de classe (Fractor et al., 1993; Frier et Vadcar, 2017; Giasson, 2011 ; Neuman et Roskos, 2008). On peut les regrouper en trois grandes catégories: ceux relatifs à l'offre, à l'aménagement et à l'accessibilité (MEESR, s. d.). Dans le cadre de cet article, nous nous intéresserons plus particulièrement à ceux relatifs à l'offre.

\section{L'offre des bibliothèques de classe}

6 Les critères qui permettent d'évaluer l'offre réfèrent à la quantité des albums ${ }^{8}$ disponibles, à leur variété ainsi qu’à leur qualité.

\section{La quantité}

7 Le nombre d'albums présents dans les classes a une incidence sur le développement de l'intérêt des enfants pour les livres et est corrélé au développement ultérieur de leur apprentissage de la lecture (Guo et al., 2012). Pour ce qui est du nombre de livres accessibles aux enfants, la bibliothèque idéale comporte au moins 8 livres par enfant (Fractor et al., 1993) et au moins 100 livres au total (Giasson et Saint-Laurent, 1999). En ce qui concerne le nombre de livres de la classe, c'est-à-dire, en incluant ceux qui peuvent être rangés, au moins 300 livres sont recommandés (Frier et Vadcar, 2017). En 
effet, afin de permettre aux enfants d'avoir accès à une plus grande quantité de livres et de maintenir leur intérêt, il est suggéré de faire une rotation des livres, au cours de l'année, ou d'ajouter des ouvrages empruntés à la bibliothèque municipale ou de l'école (Boudreau et al., 2019; Neuman et Bredekamp, 2000). Certains suggèrent de le faire deux fois par mois (Morrow, 1989), alors que d'autres recommandent de le faire une fois par mois (Tracey et Morrow, 2015).

\section{La variété}

Pour rejoindre les différents intérêts des enfants et accroître leur motivation et leur engagement à lire, il importe également de les exposer à différents genres de livres (Charron et al., accepté ; Fractor et al., 1993; Vukelich et al., 2012). À l'éducation préscolaire, les principaux genres d'albums recommandés sont le récit, l'abécédaire, le documentaire, le chiffrier, l'imagier, la comptine/poésie et l'album sans texte (Lipson et al., 2004). Les livres documentaires sont particulièrement importants, car ils permettent d'améliorer le vocabulaire des enfants, leurs connaissances du monde et leur compréhension des textes entendus (Chapman et al., 2007 ; Duke et Kays, 1998 ; Kraemer et al., 2012). Il est recommandé que la bibliothèque de classe en contienne une proportion d'au moins $30 \%$ (Moss et al., 1997).

9 Il est également suggéré d'exposer les enfants à des livres d'origines diverses. À ce sujet, le programme de formation de l'école québécoise stipule que tout au long de leur scolarité, les enfants doivent être mis en contact avec une variété de livres. Ces derniers doivent provenir d'abord de la province du Québec, puis de la francophonie ou de la littérature internationale traduite en français (MELS, 2006).

\section{La qualité}

10 La qualité des livres figurant dans une bibliothèque de classe est un critère incontournable (Lipson et al., 2004). Dans son Guide d'acquisition en ligne, le ministère de l'Éducation précise que les livres de qualité «donnent le goût de lire, stimulent l'imaginaire, soutiennent la créativité, permettent d'exercer un jugement critique et stimulent l'intelligence» (MEESR, s. d.). Pour établir des choix éclairés, il suggère de recourir à des outils de sélection comme le répertoire Constellations ${ }^{9}$ (anciennement Livres ouverts). Ce site Internet, conçu et produit par la Direction de la formation générale des jeunes (DFGJ) du ministère de l'Éducation du Québec, propose une sélection d'ouvrages de qualité destinée, notamment aux enseignants afin de les guider dans leur choix de livres pour la classe.

11 Il importe également que les ouvrages soient en bon état et récents afin de donner accès aux enfants à des albums attrayants, mais également actuels et fidèles à la réalité d'aujourd'hui (Neuman et Bredekamp 2000), ce qui n'exclut pas, bien sûr, de donner accès aux enfants à des contes patrimoniaux. Bien qu'il n'y ait pas de consensus pour déterminer la durée de vie d'un ouvrage, certaines bibliothécaires scolaires se sont dotées de balises. Par exemple, les bibliothécaires du Centre de services scolaires de Montréal considèrent qu'au-delà de 20 ans, un livre devrait être élagué, c'est-à-dire retiré de la collection (CSDM, s. d.) 


\section{Les études menées sur le sujet et les critères utilisés}

12 Si les chercheurs s'entendent sur l'importance de doter les classes de maternelle de bibliothèques de qualité et sur la façon d'y parvenir (Tracey et Morrow, 2015 ; Vukelich et al., 2012), peu de données récentes sont disponibles à propos du niveau de qualité observé sur le terrain. Du côté des études américaines, Dowhower et ses collaborateurs (1998) ont mené une enquête dans 18 classes de maternelle et se sont intéressés à la quantité de livres disponibles ainsi qu'à la variété des genres, mais en n'en considérant que trois (récit, documentaire et poésie). En outre, Guo et ses collaborateurs (2013) ont évalué la qualité des bibliothèques de classe de 54 classes de maternelle. Ils se sont, quant à eux, intéressés au nombre de livres de genres différents présents au sein des classes (récit, livre électronique, documentaire, abécédaire, poésie/comptines, livres sur des concepts, par exemple les formes ou les couleurs et les livres sur le thème actuel de la classe). Au Québec, Giasson et Saint-Laurent (1999) ont évalué la qualité des bibliothèques de classe de 29 classes de maternelle 5 ans. Dans le cadre d'un cours universitaire, Boudreau et ses collaboratrices (2019) ont repris la même démarche que Giasson pour évaluer les bibliothèques et les coins-lecture de 27 classes de maternelle 5 ans. Dans les deux derniers cas, les études se sont limitées à examiner la quantité de livres disponibles, en termes d'offre.

13 En bref, il n'existe pas, à notre connaissance, d'études récentes sur la qualité des bibliothèques de classe de maternelle québécoise, en particulier en milieu défavorisé, qui s'attacherait de façon fine au critère de l'offre, c'est-à-dire en examinant non seulement la quantité de livres disponibles, mais également leur variété et leur qualité. Considérant d'une part, l'importance pour les enfants d'âge préscolaire d'avoir accès à une bibliothèque de classe dotée d'une offre abondante, riche et variée, et d'autre part, le rôle que devrait jouer la maternelle 4 ans auprès des enfants issus de milieux défavorisés et n'ayant pas bénéficié d'un contact fréquent avec des livres de qualité, avant leur entrée à l'école, il nous semble nécessaire de décrire les bibliothèques de classe de maternelle 4 ans en milieu défavorisé et d'en évaluer le niveau de qualité.

\section{Méthodologie}

Afin de décrire les bibliothèques de classe de maternelle 4 ans à temps plein en milieu défavorisé (TPMD) québécois et d'en évaluer la qualité, nous avons mené une enquête à visée descriptive (Fortin et Gagnon, 2016).

\section{Échantillon}

Nous avons recueilli des données dans 30 classes participant à un projet de recherche de plus grande envergure dirigé par Annie Charron et portant sur la qualité de l'environnement oral et écrit dans les classes de maternelle québécoises de 4 ans en milieu défavorisé. Les écoles étaient toutes situées en milieu défavorisé (IMSE de 10 pour la majorité d'entre elles ${ }^{10}$ ) et étaient réparties dans neuf commissions scolaires à travers le Québec (régions urbaines et rurales). 


\section{Instrument et déroulement de la collecte de données}

Les données ont été recueillies à l'aide d'un outil développé pour les besoins de la recherche. Pour concevoir ce dernier, nous nous sommes appuyés sur une recension des écrits scientifiques concernant la qualité des bibliothèques de classe et avons retenu trois grands critères (offre, aménagement et accessibilité) que nous avons subdivisés en indicateurs. Toutefois, faute d'espace, nous ne présenterons que les données relatives à l'offre, dans le cadre de cet article.

L'outil est composé de formulaires à remplir en ligne à l'aide d'une tablette électronique et de l'application Device Magic. Les formulaires relatifs à l'offre permettent de recueillir des données à propos de la quantité (quantité d'albums accessibles et rangés, fréquence d'ajout et de rotation), de la variété (pour chacun des albums : genre, origine, langue, langue originale) et de la qualité (provenance et, pour chacun des albums : titre, auteur, éditeur, année de publication et condition).

Les formulaires ont été remplis par des auxiliaires de recherche formées à leur utilisation qui ont visité les classes au printemps 2019. Chaque album accessible aux enfants, le jour de la collecte de données, a fait l'objet d'une entrée dans l'outil : titre, auteur, éditeur, genre, année de publication, origine, langue et état. Les auxiliaires ont également pris une photographie de la page de couverture de chacun des albums répertoriés et des bibliothèques de classe. Pour finir, ils ont fait un court entretien avec chaque enseignant afin de compléter la collecte de données (par exemple, à propos de la fréquence de rotation ou d'ajout d'albums et de la présence d'un budget spécial pour l'achat de livres dans l'année courante).

\section{Analyse des données}

9 Afin de pouvoir dresser le portrait des bibliothèques de classe relativement à l'offre, nous avons associé des échelles descriptives aux trois indicateurs du critère (quantité, variété, qualité). Les données quantitatives ont été compilées et traitées à l'aide de statistiques descriptives afin de dégager un portait du corpus d'ensemble et une description plus détaillée de la situation observée dans chacune des classes (fréquence, pourcentage et moyenne).

20 Finalement, afin d'attribuer un score de qualité globale aux bibliothèques de chacune des classes, nous avons également développé une grille d'évaluation avec une échelle descriptive à quatre niveaux (allant de 0 à 2 points, avec un intervalle de 0,5 ) pour chacun des indicateurs de l'offre (quantité, variété et qualité).

\section{Résultats}

21 Afin de décrire les bibliothèques de classe, nous présentons d'abord un portrait du corpus des albums répertoriés dans les bibliothèques des 30 classes de maternelle 4 ans TPMD. Puis, dans un deuxième temps, nous présentons un portait des bibliothèques de classe à l'aide de chacun des indicateurs du critère de l'offre, c'est-à-dire ceux relatifs à la quantité, à la variété et à la qualité. Enfin, afin d'évaluer le niveau de qualité global des bibliothèques de classe, toujours au regard des indicateurs de l'offre, nous présentons des données relatives aux scores obtenus par les 30 classes. 


\section{Portrait du corpus des albums répertoriés dans les bibliothèques de classe}

\section{Toutes les}

\section{Quantité d'albums}

Quantité d'albums accessibles aux enfants

Tous les albums qui étaient accessibles aux enfants le jour de la collecte de données (ce qui exclut les albums rangés, par exemple, dans une armoire fermée) ont été répertoriés pour un total de 2539 .

\section{Quantité totale d'albums de la classe}

Le portrait de la quantité d'albums auxquels les enfants ont accès ne peut être complet sans prendre en compte les livres qui sont rangés (non accessibles aux enfants, mais qui peuvent faire l'objet de rotation, au cours de l'année). Pour en tenir compte, nous avons donc également dénombré ces albums. En additionnant ces deux nombres, cela mène à un total de 8054 albums dans l'ensemble des classes, ce qui signifie que les enfants n'avaient accès, en moyenne, qu'à $31,5 \%$ de la collection d'albums appartenant à la classe, le jour de la collecte de données. Pour des raisons de faisabilité, nous avons choisi de limiter notre collecte de données détaillées (titre, auteur, éditeur, genre, année de publication, origine, langue et condition) ainsi que nos analyses aux titres accessibles aux enfants, le jour de notre collecte de données, soit 2539 albums.

\section{Variété des albums du corpus}

Les albums du corpus répertorié se présentent sous une variété de genres, de langues et de pays d'origine.

\section{Genres des albums répertoriés}

Sur les 2539 albums du corpus, le récit est le genre le plus répertorié $(68,4 \%)$ suivi, dans des proportions plus modestes, du documentaire $(13,2 \%)$ et de l'imagier $(6,9 \%)$. La catégorie " autre » qui regroupe, par exemple, les livres-jeu, les cahiers d'autocollants et les livres de type «cherche et trouve » représente, quant à elle, 6,5\%. Dans de très faibles proportions suivent les chiffriers $(1,7 \%)$, les albums de comptines/poésie $(1,4 \%)$, les abécédaires $(1,3 \%)$ et les albums sans texte $(0,6 \%)$. La figure 1 présente la distribution des albums accessibles dans les bibliothèques de classe en fonction du genre. 
Figure 1 - Distribution des albums en fonction du genre

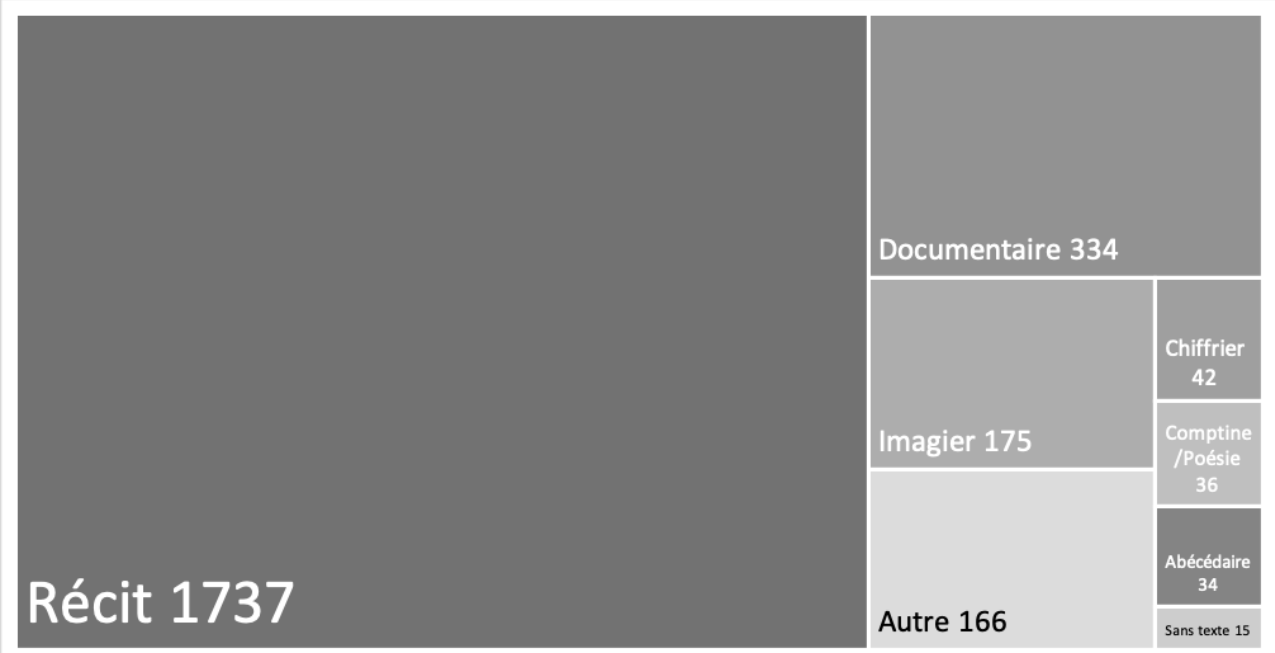

\section{Langue des albums répertoriés}

La quasi-totalité des 2539 albums (98,7 \%) est publiée en français (figure 2). Cependant, si un peu moins des deux tiers des albums sont en français langue originale $(60,1 \%)$, un peu plus du tiers ont été traduits d'une autre langue (38,6\%). Une faible proportion d'albums est publiée en langue anglaise (1,3\%). Aucun album publié dans une autre langue que le français ou l'anglais n'a été répertorié.

Figure 2 - Langue des albums

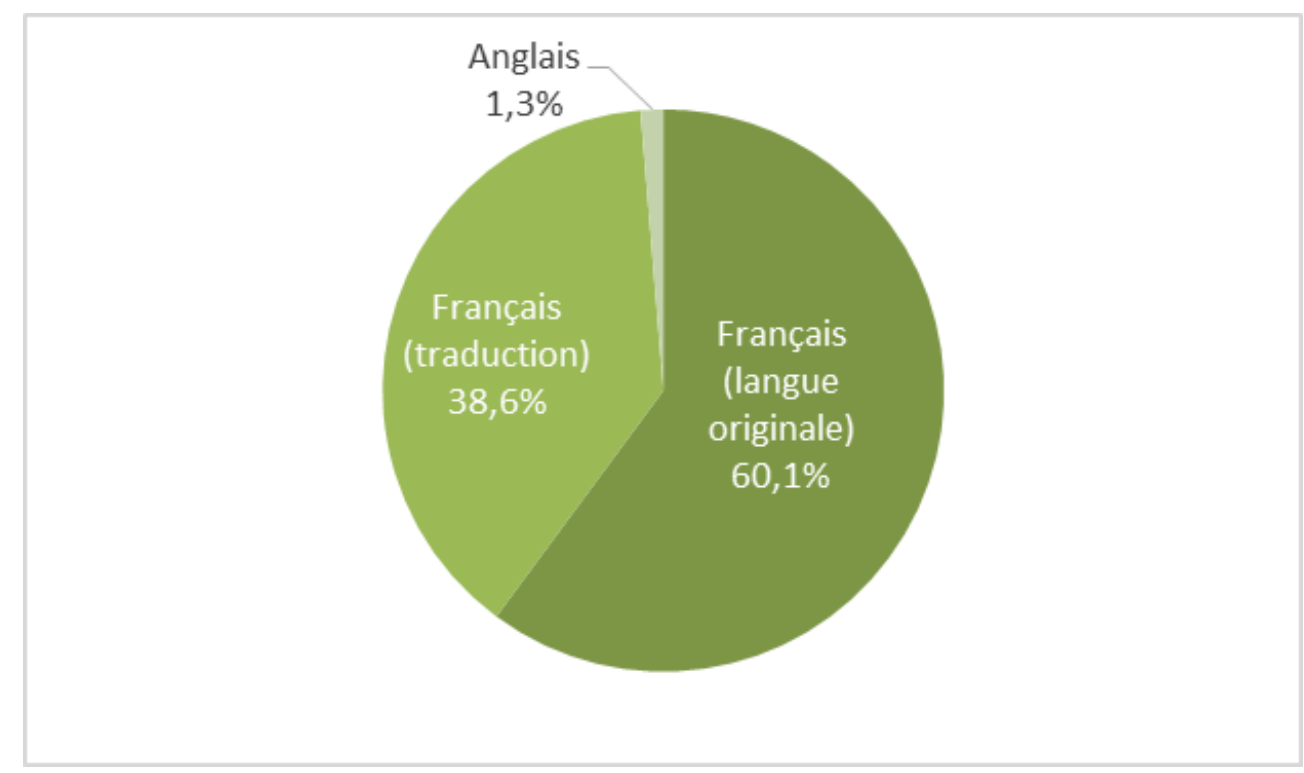

\section{Lieu d'origine des albums répertoriés}

En ce qui concerne l'origine des albums répertoriés, une forte proportion des albums présents en classe provient de pays européens (44,5\%) ou du Québec (40,0\%). Dans une moindre mesure, on retrouve des albums originaires du reste du Canada $(10,3 \%)$ et des États-Unis (2,5\%). Soulignons toutefois qu'en excluant les albums jeunesse publiés par 
Phidal et Grolier ${ }^{11}$ (deux éditeurs québécois qui ne publient pas de contenu original, mais plutôt des albums commerciaux pour des compagnies comme Mattel ou Marvel), la proportion d'albums d'éditeurs québécois passe de $40 \%$ à $27,1 \%$. La figure 3 illustre la façon dont les albums se répartissent selon le lieu d'origine de leur maison d'édition.

Figure 3 - Lieu d'origine des albums

*Données manquantes: aucun lieu d'origine ne figurait dans l'album.

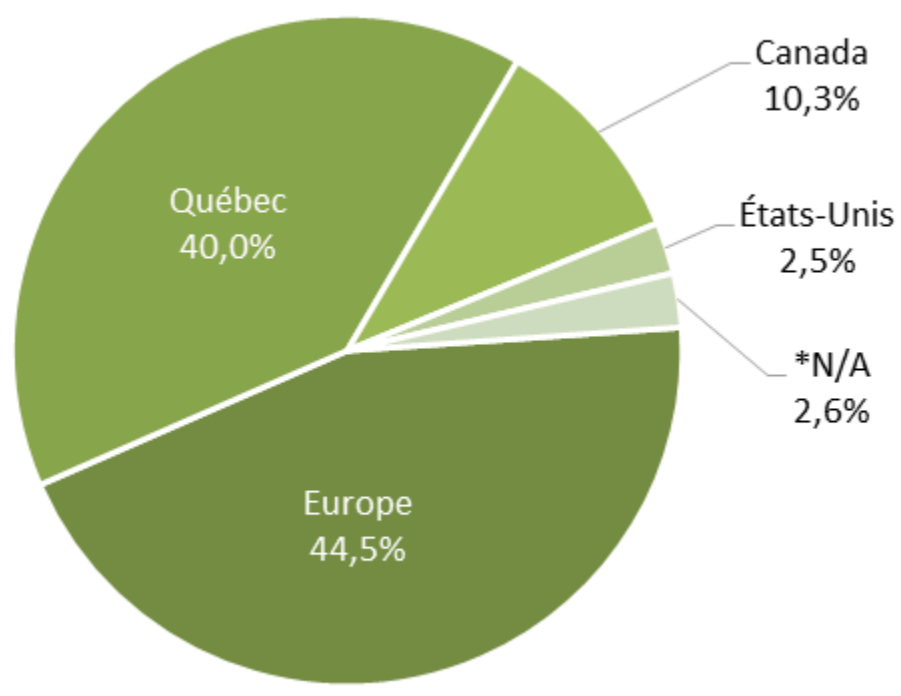

Qualité des albums du corpus

Afin de pouvoir décrire la qualité des albums présents au sein du corpus, nous nous sommes appuyés sur la légitimité de l'éditeur dans le réseau institutionnalisé du livre, sur leur présence dans le répertoire Constellations, leur année de publication ainsi que leur état.

\section{Légitimité accordée à l'éditeur}

Dans le but d'affiner notre analyse en ce qui concerne la qualité des albums du corpus, nous avons retenu le critère de légitimité de l'éditeur dans le réseau institutionnalisé du livre, c'est-à-dire leur reconnaissance. Nous avons ainsi distingué les éditeurs dits "reconnus ", c'est-à-dire ceux qui vendent leurs ouvrages en librairie (par exemple, l'éditeur québécois La courte échelle ou l'éditeur français L'École des loisirs) de ceux de type plus commercial qui vendent surtout dans les grandes surfaces ou les magasins d'aubaines (par exemple, Disney ou Phidal).

31 Sur la base de cette distinction, 63,7 \% des albums présents dans les bibliothèques de classe sont publiés par des éditeurs dits « reconnus », alors que $36,3 \%$ ne le sont pas. 


\section{Présence dans le répertoire Constellations} le répertoire Constellations. Rappelons que ce répertoire propose une sélection d'ouvrages de qualité, destinée notamment aux enseignants, afin de les guider dans leur choix de livres pour la classe.

\section{Année de publication des albums}

En ce qui concerne l'année de publication des albums (tableau 1), parmi les 2539 albums recensés, une majorité d'ouvrages a été publiée à partir de l'an 2000 (70,2\%).

Tableau 1 Année de publication des albums

* Données manquantes : aucune date ne figurait dans l'album.

\begin{tabular}{|l|l|l|}
\hline Année de publication & $n$ & $\%$ \\
\hline $1940-1989$ & 230 & 9,1 \\
\hline $1990-1999$ & 470 & 18,5 \\
\hline $2000-2009$ & 773 & 30,4 \\
\hline $2010-2019$ & 1010 & 39,8 \\
\hline N/A* & 56 & 2,2 \\
\hline Total & 2539 & 100 \\
\hline
\end{tabular}

\section{État des albums}

Afin de déterminer l'état physique de chaque album, un examen visuel a été effectué pour repérer des déchirures, des taches, des pages pliées, de l'usure et de l'écriture sur les albums jeunesse. Chaque album a ensuite été catégorisé comme étant soit «très abimé ", " abimé », " un peu abimé » ou " en parfait état » (figure 4). La majorité des albums répertoriés sont considérés soit comme " un peu abimés » ou " en parfait état " $(62,6 \%)$. Les albums jugés « abimés » forment, quant à eux, $24,7 \%$ de la sélection et les albums « très abimés » seulement $12,7 \%$. 


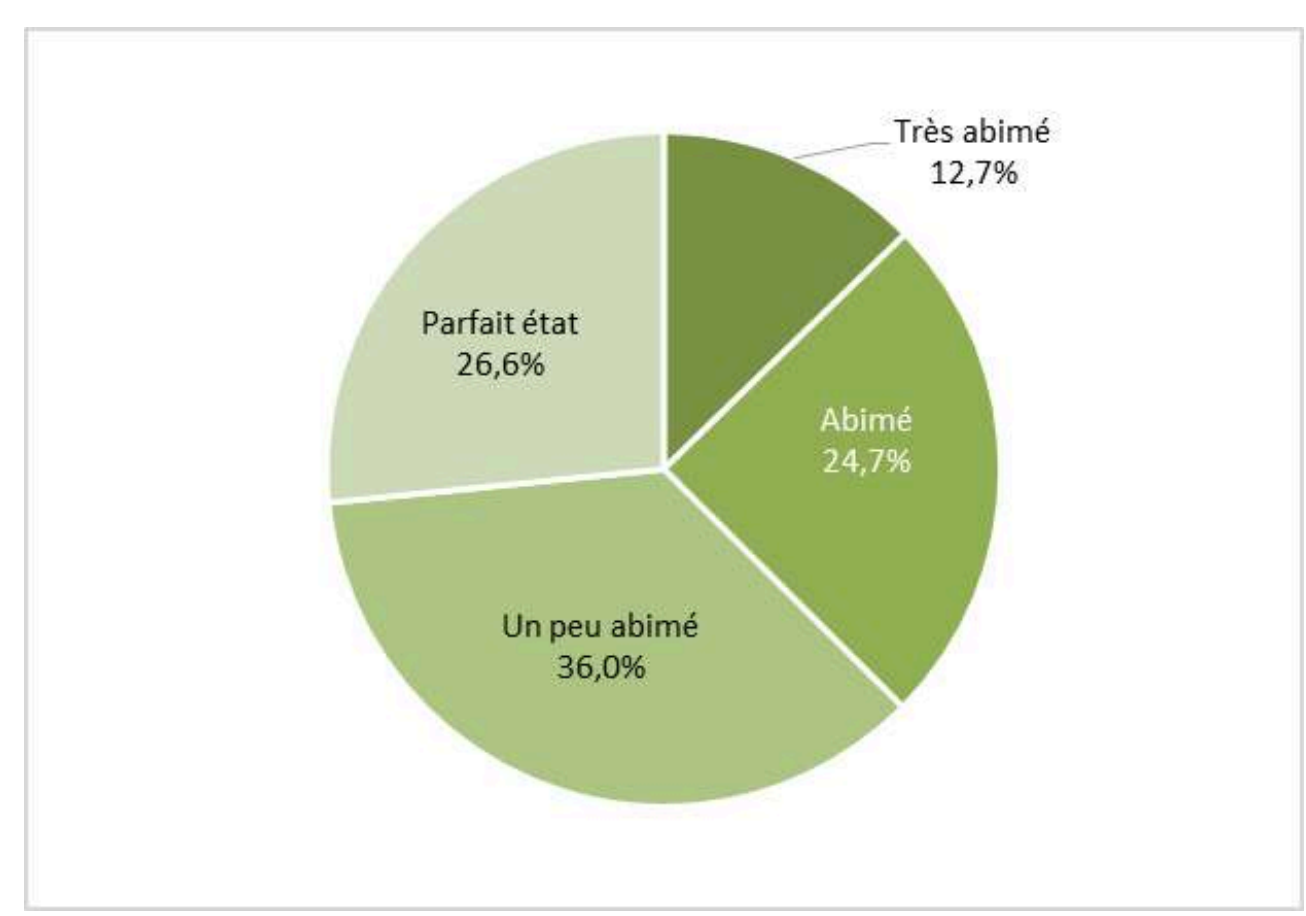

\section{Portrait des bibliothèques de classe}

Afin de pouvoir décrire les bibliothèques de classe en ce qui concerne l'offre, nous avons associé des échelles descriptives à ces trois indicateurs (quantité, variété, qualité).

\section{La quantité d'albums}

Le tableau 2 présente les données détaillées relatives au critère de la quantité d'albums accessibles aux enfants (indicateur 1.1). Le jour de la collecte, seulement $30 \%$ des classes en ont au moins 100. La majorité (70\%) en a moins de 75. Les classes ont, en moyenne, 84,6 albums accessibles aux enfants, le jour de la collecte ; la classe en ayant le moins en compte seulement 17 , alors que celle en ayant le plus en compte 282 .

Pour ce qui a trait au nombre d'albums accessibles par enfant (indicateur 1.2), les bibliothèques en comptent, en moyenne, 6,8 par enfant. Si toutes les classes offrent au moins un album par enfant, la majorité des classes (56,7\%) en ont moins de 4 par enfant et seulement $30 \%$ en ont au moins 8 par enfant.

La majorité des enseignants déclarent effectuer la rotation de leurs albums (indicateur 1.3) une fois par mois (43,3\%) ou quelques fois par mois (33,3\%). Seul un petit nombre d'enseignants déclarent faire la rotation des albums de leur bibliothèque de classe une fois par semaine $(13,3 \%)$ et un seul enseignant $(3,3 \%)$ déclare ne jamais faire de rotation des albums.

Quant à l'ajout d'albums empruntés à la bibliothèque municipale ou de l'école (indicateur 1.4), une grande proportion des enseignants déclare ajouter des albums jeunesse à leur bibliothèque de classe quelques fois par mois (43,3\%). Un plus petit nombre d'entre eux déclarent en ajouter une fois par mois $(20 \%)$ ou quelques fois par 
année (13,3\%). Trois enseignants (10\%) déclarent ne jamais en ajouter tandis que quatre enseignants déclarent en ajouter jusqu'à une fois par semaine (13,3 \%).

Tableau 2 La distribution des classes concernant l'offre : quantité

\begin{tabular}{|c|c|c|c|c|c|}
\hline $\begin{array}{l}1.1 \text { Quantité d'albums } \\
\text { accessibles }\end{array}$ & $\begin{array}{l}\text { Au moins } \\
100\end{array}$ & $\begin{array}{l}\text { De } 75 \\
\text { à } 99\end{array}$ & $\begin{array}{l}\text { De } 50 \\
\text { à } 74\end{array}$ & $\begin{array}{l}\text { De } 25 \\
\text { à } 49\end{array}$ & $\begin{array}{l}\text { Moins } \\
\text { de } 25\end{array}$ \\
\hline $\begin{array}{l}n \\
\%\end{array}$ & $\begin{array}{l}9 \\
30\end{array}$ & $\begin{array}{l}0 \\
0\end{array}$ & $\begin{array}{l}5 \\
16,7\end{array}$ & $\begin{array}{l}12 \\
40\end{array}$ & $\begin{array}{l}4 \\
13,3\end{array}$ \\
\hline $\begin{array}{l}\text { Nombre d'albums moyen par } \\
\text { classe }\end{array}$ & 84,6 & & & & \\
\hline $\begin{array}{l}1.2 \text { Quantité d'albums } \\
\text { accessibles par enfant }\end{array}$ & $\begin{array}{l}\text { Au moins } \\
8\end{array}$ & $\begin{array}{l}\text { De } 6 \\
\text { à } 7,9\end{array}$ & $\begin{array}{l}\text { De } 4 \\
\text { à } 5,9\end{array}$ & $\begin{array}{l}\text { De } 1 \\
\text { à } 3,9\end{array}$ & $\begin{array}{l}\text { Moins } \\
\text { de } 1\end{array}$ \\
\hline $\begin{array}{l}n \\
\%\end{array}$ & $\begin{array}{l}9 \\
30\end{array}$ & $\begin{array}{l}0 \\
0\end{array}$ & $\begin{array}{l}4 \\
13,3\end{array}$ & $\begin{array}{l}17 \\
56,7\end{array}$ & $\begin{array}{l}0 \\
0\end{array}$ \\
\hline $\begin{array}{l}\text { Nombre moyen d'albums par } \\
\text { enfant par classe }\end{array}$ & 6,8 & & & & \\
\hline $\begin{array}{l}\text { 1.3 Fréquence de la rotation } \\
\text { des albums }\end{array}$ & $\begin{array}{l}\text { Une fois } \\
\text { par } \\
\text { semaine }\end{array}$ & $\begin{array}{l}\text { Quelques } \\
\text { fois par } \\
\text { mois }\end{array}$ & $\begin{array}{l}\text { Une fois } \\
\text { par } \\
\text { mois }\end{array}$ & $\begin{array}{l}\text { Quelques } \\
\text { fois par } \\
\text { année }\end{array}$ & Jamais \\
\hline $\begin{array}{l}n \\
\%\end{array}$ & $\begin{array}{l}4 \\
13,3\end{array}$ & $\begin{array}{l}10 \\
33,3\end{array}$ & $\begin{array}{l}13 \\
43,3\end{array}$ & $\begin{array}{l}2 \\
6,7\end{array}$ & $\begin{array}{l}1 \\
3,3\end{array}$ \\
\hline $\begin{array}{l}\text { 1.4 Fréquence d'ajout d'albums } \\
\text { (emprunts bibliothèque } \\
\text { municipale ou de l'école) }\end{array}$ & $\begin{array}{l}\text { Une fois } \\
\text { par } \\
\text { semaine }\end{array}$ & \begin{tabular}{|l} 
Quelques \\
fois par \\
mois
\end{tabular} & $\begin{array}{l}\text { Une fois } \\
\text { par } \\
\text { mois }\end{array}$ & $\begin{array}{l}\text { Quelques } \\
\text { fois par } \\
\text { année }\end{array}$ & Jamais \\
\hline $\begin{array}{l}n \\
\%\end{array}$ & $\begin{array}{l}4 \\
13,3\end{array}$ & $\begin{array}{l}13 \\
43,3\end{array}$ & $\begin{array}{l}6 \\
20\end{array}$ & $\begin{array}{l}4 \\
13,3\end{array}$ & $\begin{array}{l}3 \\
10\end{array}$ \\
\hline
\end{tabular}

\section{La variété des albums}

Alors que les données précédentes offrent une indication quant à la quantité d'albums à laquelle les enfants sont exposés, elles renseignent peu sur les différents genres et origines qui les caractérisent. C'est pourquoi le tableau 3 présente également des données détaillées relatives au critère de variété.

41 En ce qui concerne la variété de genres (indicateur 2.1), en excluant les albums figurant dans la catégorie " autre » (c'est-à-dire, celle qui regroupe, par exemple, les livres-jeu, les cahiers d'autocollants et les livres de type "cherche et trouve »), la majorité des classes $(53,4 \%)$ a des albums appartenant à 5 à 7 genres différents, plus du quart 
$(26,7 \%)$ en ont 4 , tandis qu'un plus petit nombre n'en ont que 3 ou 2 (respectivement $16,7 \%$ et $3,3 \%)$. On compte en moyenne 4,7 genres d'albums par classe.

Parmi les 30 classes de l'échantillon, le pourcentage de documentaires (indicateur 2.2) est relativement faible. En effet, seulement une classe $(3,3 \%)$ possède au moins $30 \%$ de documentaires. Le tiers (33,3\%) en possèdent moins de $10 \%$. En moyenne, on trouve $15,2 \%$ de documentaires dans les classes de l'échantillon, la classe en ayant le moins en comptant $2,3 \%$ et celle en ayant le plus en comptant $40 \%$.

Pour ce qui est de la variété d'origines (indicateur 2.3), la majorité des classes (90\%) ont des albums qui présentent une variété (au moins 3), tandis que seulement 3 classes (10\%) ont des albums qui ne proviennent que de deux origines différentes.

Concernant le pourcentage d'ouvrages québécois (indicateur 2.4), sur les 30 classes de l'échantillon, seulement 6 d'entre elles (20\%) ont au moins $50 \%$ d'ouvrages québécois. Toutes les classes comptent toutefois des albums d'éditeurs québécois dans leur bibliothèque de classe, dans une proportion allant de $13,3 \%$ à $71,7 \%$, la proportion moyenne par classe s'établissant à 34,8\%.

Tableau 3 La distribution des classes concernant l'offre : variété

\begin{tabular}{|c|c|c|c|c|c|}
\hline 2.1 Variété de genres & $\begin{array}{l}\text { Présence } \\
\text { de 6-7 }\end{array}$ & $\begin{array}{l}\text { Présence } \\
\text { de } 5\end{array}$ & $\begin{array}{l}\text { Présence } \\
\text { de } 4\end{array}$ & $\begin{array}{l}\text { Présence } \\
\text { de } 3\end{array}$ & $\begin{array}{l}\text { Présence de } \\
2\end{array}$ \\
\hline$n$ & 8 & 8 & 8 & 5 & 1 \\
\hline$\%$ & 26,7 & 26,7 & 26,7 & 16,7 & 3,3 \\
\hline $\begin{array}{l}\text { Nombre moyen de genres par } \\
\text { classe }\end{array}$ & 4,7 & & & & \\
\hline $\begin{array}{l}2.2 \quad \text { Pourcentage de } \\
\text { documentaires }\end{array}$ & $\begin{array}{l}\text { Au moins } \\
30 \%\end{array}$ & $\begin{array}{lll}\text { De } \quad 24 & \text { à } \\
29 \% & \end{array}$ & $\begin{array}{lll}\text { De } & 17 & \text { à } \\
23 & \% & \end{array}$ & $\begin{array}{lll}\text { De } & 10 & \text { à } \\
16 \% & \end{array}$ & $\begin{array}{l}\text { Moins de } \\
10 \%\end{array}$ \\
\hline $\begin{array}{l}n \\
\%\end{array}$ & $\begin{array}{l}1 \\
3,3\end{array}$ & $\begin{array}{l}6 \\
20\end{array}$ & $\begin{array}{l}5 \\
16,7\end{array}$ & $\begin{array}{l}8 \\
26,7\end{array}$ & $\begin{array}{l}10 \\
33,3\end{array}$ \\
\hline $\begin{array}{l}\text { Pourcentage moyen de } \\
\text { documentaires } \\
\text { par classe }\end{array}$ & 15,2 & & & & \\
\hline 2.3 Variété d'origine & $\begin{array}{l}\text { Présence } \\
\text { de } 3\end{array}$ & - & $\begin{array}{l}\text { Présence } \\
\text { de } 2\end{array}$ & - & $\begin{array}{l}1 \quad \text { seule } \\
\text { présente }\end{array}$ \\
\hline $\begin{array}{l}n \\
\%\end{array}$ & $\begin{array}{l}27 \\
90\end{array}$ & & $\begin{array}{l}3 \\
10\end{array}$ & & $\begin{array}{l}0 \\
0\end{array}$ \\
\hline $\begin{array}{l}2.4 \text { Pourcentage d'albums } \\
\text { québécois }\end{array}$ & $\begin{array}{l}\text { Au moins } \\
50 \%\end{array}$ & $40-49 \%$ & $26 \%-39 \%$ & $10-25 \%$ & $\begin{array}{ll}\text { Moins de } \\
10 \%\end{array}$ \\
\hline
\end{tabular}




\begin{tabular}{|l|l|l|l|l|l|}
\hline $\boldsymbol{n}$ & 6 & 4 & 10 & 10 & 0 \\
\hline & 20 & 13,3 & 33,3 & 33,3 & 0 \\
\hline $\begin{array}{l}\text { Pourcentage moyen d'albums } \\
\text { québécois par classe }\end{array}$ & 34,8 & & & & \\
\hline
\end{tabular}

\section{La qualité des albums}

Le tableau 4 présente les données détaillées relatives au critère de qualité. Pour ce qui a trait à la légitimité accordée à l'éditeur (indicateur 3.1), dans un peu plus du tiers des classes (36,7\%), au moins $80 \%$ des albums sont publiés par des éditeurs dits "reconnus ». La moyenne d'albums publiés par des éditeurs dits "reconnus» par classe est de 69,7\%. La classe en ayant le moins affiche un taux de 33,7\%, alors que celle en ayant le plus comporte $100 \%$ d'albums publiés par de tels éditeurs.

Parmi les 30 classes, aucune ne possède de collection contenant plus de $40 \%$ d'albums recommandés par le site Constellations (indicateur 3.2). Seulement 1 classe (3,3\%) détient une collection, dont 26 à $40 \%$ des albums sont recommandés par ce site. Plus du tiers des classes $(36,7 \%)$ en ont une qui n'en comprend que de 10 à $25 \%$ et une majorité de classes $(60 \%)$ possède une collection dont moins de $10 \%$ sont répertoriés par le site Constellations. En moyenne, par classe, ce sont 9,5\% des albums qui sont recommandés par Constellations, la classe en ayant le plus en proportion en comptant $26,3 \%$ et celles en ayant le moins, $0 \%$.

Pour ce qui est de l'âge de la collection d'albums au sein de chaque classe (indicateur 3.3), la majorité des classes (60\%) détient au moins $80 \%$ d'albums publiés à partir de l'an 2000. En moyenne, $71,8 \%$ des albums accessibles ont été publiés entre 2000 et 2019 dans une proportion allant de $46,1 \%$ à 96,9\%.

Enfin, en ce qui concerne l'état des albums (indicateur 3.4), une majorité de classes $(53,3 \%)$ possède une collection dont $60 \%$ ou plus des albums sont en parfait état ou un peu abimés. Le pourcentage moyen des albums en parfait état ou un peu abimés par classe est de $63,4 \%$.

Tableau 4 La distribution des classes concernant l'offre : qualité

\begin{tabular}{|l|l|l|l|l|l|}
\hline $\begin{array}{l}\text { 3.1 Légitimité de l'éditeur } \\
\text { (pourcentage d'albums publiés par des } \\
\text { éditeurs dits « reconnus ») }\end{array}$ & $\begin{array}{l}\text { Au } \\
\text { moins } \\
80 \%\end{array}$ & $\begin{array}{l}\text { De } \\
60 \\
\text { à } \\
79 \%\end{array}$ & $\begin{array}{l}\text { De 40 \% } \\
\text { à } 59 \%\end{array}$ & $\begin{array}{l}\text { De 20 \% } \\
\text { à } 39 \%\end{array}$ & $\begin{array}{l}\text { Moins de } \\
20 \%\end{array}$ \\
\hline$n$ & 11 & 10 & 6 & 3 & 0 \\
$\%$ & 36,7 & 33,3 & 20 & 10 & 0 \\
\hline $\begin{array}{l}\text { Pourcentage moyen d'albums publiés par } \\
\text { des éditeurs dits « reconnus " par classe }\end{array}$ & 69,7 & & & & \\
\hline
\end{tabular}




\begin{tabular}{|c|c|c|c|c|c|}
\hline $\begin{array}{l}\text { 3.2 Présence dans Constellations } \\
\text { (pourcentage d'albums recommandés) }\end{array}$ & $\begin{array}{l}\mathrm{Au} \\
\text { moins } \\
50 \%\end{array}$ & $\begin{array}{l}\text { De } \\
41 \% \\
\text { à } \\
49 \%\end{array}$ & $\begin{array}{l}\text { De } 26 \% \\
\text { à } 40 \%\end{array}$ & $\begin{array}{l}\text { De } 10 \% \\
\text { à } 25 \%\end{array}$ & $\begin{array}{l}\text { Moins de } \\
10 \%\end{array}$ \\
\hline$n$ & 0 & 0 & 1 & 11 & 18 \\
\hline$\%$ & 0 & 0 & 3,3 & 36,7 & 60 \\
\hline $\begin{array}{l}\text { Pourcentage moyen d'albums recommandés } \\
\text { par classe }\end{array}$ & 9,5 & & & & \\
\hline $\begin{array}{l}\text { 3.3 Année de publication (pourcentage } \\
\text { d'albums publiés à partir de l'an 2000) }\end{array}$ & $\begin{array}{l}\mathrm{Au} \\
\text { moins } \\
80 \%\end{array}$ & \begin{tabular}{|l} 
De \\
$60 \%$ \\
à \\
$79 \%$
\end{tabular} & $\begin{array}{l}\text { De } 40 \% \\
\text { à } 59 \%\end{array}$ & $\begin{array}{l}\text { De } 20 \% \\
\text { à } 39 \%\end{array}$ & $\begin{array}{l}\text { Moins de } \\
20 \%\end{array}$ \\
\hline$n$ & 18 & 7 & 5 & 0 & 0 \\
\hline$\%$ & 60 & 23,3 & 16,7 & 0 & 0 \\
\hline $\begin{array}{l}\text { Pourcentage moyen d'albums publiés à } \\
\text { partir de l'an } 2000 \text { par classe }\end{array}$ & 71,8 & & & & \\
\hline $\begin{array}{l}\text { 3. } 4 \text { Condition } \\
\text { (pourcentage d'albums en parfait état ou un } \\
\text { peu abimés) }\end{array}$ & $\begin{array}{l}\mathrm{Au} \\
\text { moins } \\
80 \%\end{array}$ & $\begin{array}{l}\text { De } \\
60 \% \\
\text { à } \\
79 \%\end{array}$ & $\begin{array}{l}\text { De } 40 \% \\
\text { à } 59 \%\end{array}$ & $\begin{array}{l}\text { De } 20 \% \\
\text { à } 39 \%\end{array}$ & $\begin{array}{l}\text { Moins de } \\
20 \%\end{array}$ \\
\hline $\begin{array}{l}n \\
\%\end{array}$ & $\begin{array}{l}8 \\
26,7\end{array}$ & $\begin{array}{l}8 \\
26,7\end{array}$ & $\begin{array}{l}10 \\
33,3\end{array}$ & \begin{tabular}{|l}
4 \\
13,3
\end{tabular} & $\begin{array}{l}0 \\
0\end{array}$ \\
\hline $\begin{array}{l}\text { Pourcentage moyen d'albums en parfait état } \\
\text { ou un peu abimés par classe }\end{array}$ & 63,4 & & & & \\
\hline
\end{tabular}

\section{Qualité globale des bibliothèques de classe pour ce qui a trait à l'offre}

Finalement, afin d'évaluer la qualité globale des bibliothèques de classe pour ce qui a trait à l'offre, un score a été calculé pour chaque bibliothèque en fonction du nombre de points attribué ( 2 pts, 1,5 pts, 1 pt, 0,5 pt ou 0 pt) à chacun des indicateurs présentés plus haut (quantité, variété, qualité). Par exemple, concernant l'état des livres, 0 point était attribué à une classe ayant moins de $20 \%$ d'albums en parfait état ou un peu abimés tandis que 2 points étaient attribués à celle en ayant au moins $80 \%$. Le tableau 5 présente la fréquence et le pourcentage relatif à chaque niveau de qualité observé.

Tableau 5 La distribution des niveaux de qualité observés des bibliothèques de classe (relativement au critère de l'offre seulement)

\begin{tabular}{|l|l|l|l|l|l|l|}
\hline $\begin{array}{l}21,7 \text { à } 24 \\
\text { pts }\end{array}$ & $\begin{array}{l}19,3 \\
\text { pts à }\end{array}$ & 21,6 & $\begin{array}{l}16,9 \text { à } 19,2 \\
\text { pts }\end{array}$ & 14,5 à 16,8 pts & $\begin{array}{l}12,1 \quad \text { à } 14,4 \\
\text { pts }\end{array}$ & $\begin{array}{l}0 \text { à } 12 \\
\text { pts }\end{array}$ \\
\hline
\end{tabular}




\begin{tabular}{|c|c|c|c|c|c|c|}
\hline & $\begin{array}{l}\text { Excellent } \\
\text { niveau de } \\
\text { qualité }\end{array}$ & $\begin{array}{lr}\text { Très } & \text { bon } \\
\text { niveau } & \text { de } \\
\text { qualité } & \end{array}$ & $\begin{array}{l}\text { Bon niveau } \\
\text { de qualité }\end{array}$ & $\begin{array}{l}\text { Niveau } \\
\text { minimal de } \\
\text { qualité }\end{array}$ & $\begin{array}{l}\text { Faible niveau } \\
\text { de qualité }\end{array}$ & $\begin{array}{lr}\text { Très } & \text { faible } \\
\text { niveau } & \text { de } \\
\text { qualité } & \end{array}$ \\
\hline$n$ & 0 & 2 & 3 & 10 & 10 & 5 \\
\hline$\%$ & 0 & 6,7 & 10 & 33,3 & 33,3 & 16,7 \\
\hline
\end{tabular}

Sur une possibilité de 24 points, le score attribué à chaque bibliothèque varie de 9 à 20,5 points pour une moyenne de 14,5 points. La majorité d'entre elles $(83,3 \%)$ obtiennent un score qui ne dépasse pas le seuil minimal et la moitié d'entre elles (50\%) se situent sous le seuil minimal. Un petit nombre d'entre elles (10\%) obtiennent un score qui leur accorde un bon niveau de qualité et seul un très petit nombre d'entre elles $(6,7 \%)$ obtiennent un score qui leur accorde un très bon niveau. Aucune des 30 bibliothèques n'obtient un niveau de qualité excellent. Dans l'ensemble, il ressort que le niveau de la qualité des bibliothèques, en ce qui concerne l'offre, se situe entre le niveau faible et minimal, et ce, autant pour le critère de la quantité, que celui de la variété et de la qualité.

\section{Discussion}

51 L'objectif de notre recherche était de décrire les bibliothèques de classe et d'en évaluer la qualité. Dans le cadre de cet article, nous avons présenté les résultats relatifs au critère de l'offre (quantité, variété et qualité).

\section{La quantité d'albums}

En ce qui concerne le critère de la quantité, nos résultats montrent qu'une grande quantité des albums sont rangés : les enfants n'avaient accès qu'à $31,5 \%$ de la collection d'albums appartenant à la classe, le jour de la collecte de données. Près du tiers des classes (30\%) ont au moins 100 albums et seulement 13,3\% en ont moins de 25 . Ces données sont encourageantes lorsqu'on les compare à celles obtenues dans l'étude de Giasson et Saint-Laurent (1999) où aucune des 29 classes de l'étude n'avait plus de 100 albums et $66,7 \%$ des classes en avaient 25 et moins. Une vingtaine d'années séparant ces deux études, il est possible d'avancer que les diverses mesures gouvernementales des dernières années, dont le Plan d'action en lecture, mis en œuvre de 2005 à 2011, ont encouragé l'achat de livres pour les classes. Il faut toutefois souligner que, malgré cette amélioration, le nombre d'albums répertoriés dans chaque classe se situe en deçà des recommandations pour la grande majorité d'entre elles (70\%). En ce qui concerne le nombre d'albums accessibles par enfant, la majorité des classes (56,7\%) en avait de 1 à 3,9 , ce qui se rapproche des résultats observés dans l'étude de Boudreau et ses collaboratrices (2019) où $53 \%$ des 27 classes de l'échantillon avaient de 1 à 3 livres par enfants. Toutefois, là où nos résultats se distinguent, c'est en ce qui concerne le pourcentage de classes ayant au moins 8 albums par enfant qui atteint $30 \%$ dans notre étude alors qu'il n'atteint que $7 \%$ dans l'étude de Boudreau et al. Par ailleurs, nos résultats montrent que presque tous les enseignants procèdent à la rotation et à l'ajout d'albums empruntés à la bibliothèque municipale ou de l'école, ce qui permet de croire, 
qu'au cours de l'année, les enfants ont donc accès à un nombre encore plus grand de livres.

\section{La variété}

53 Pour ce qui a trait à la variété des genres présents au sein des bibliothèques de classe, la majorité d'entre elles ( $80 \%$ ) ont une variété de genres ( 4 et plus) dans leur bibliothèque de classe, ce qui peut être attribuable au grand nombre d'albums présents en classe. Toutefois, cette variété est asymétrique puisque le récit domine et représente $68,4 \%$ des albums du corpus tandis que le documentaire, le deuxième genre le plus présent, n'en représente que 13,2\%. Ces résultats sont similaires à ceux obtenus par Dowhower et ses collaborateurs (1998) et qui avaient observé que $92,5 \%$ du corpus des bibliothèques de classe étaient constitués de récits et que seulement $5,33 \%$ étaient des documentaires. À l'instar de Colman (2007), nous pouvons avancer deux hypothèses pour justifier la faible présence des documentaires dans les bibliothèques de classe : le prix plus élevé de ces derniers et le fait que les enseignants préfèrent généralement les histoires. Pourtant, les albums documentaires devraient occuper une place de choix dans les bibliothèques de classe, et ce, en raison de leur potentiel pour enrichir le vocabulaire et la compréhension du monde (Chapman et al., 2007). D'autres genres très importants pour le développement des jeunes enfants sont également présents en trop petit nombre dans les bibliothèques de classe : les chiffriers $(1,7 \%)$, les abécédaires $(1,3 \%)$ et les livres de poésie-comptines $(1,4 \%)$. Il est également préoccupant que la catégorie "autre " (c'est-à-dire, celle qui regroupe, par exemple, les livres-jeu, les cahiers d'autocollants et les livres de type "cherche et trouve»), soit, en moyenne, 4 fois plus présente au sein du corpus $(6,5 \%)$ que chacun de ces genres.

Si la quasi-totalité des albums sont des ouvrages en français, 38,6\% d'entre eux ont été traduits d'une autre langue et $1,3 \%$ sont en anglais. Aucun album publié dans une autre langue que le français ou l'anglais n'a été répertorié. Ce résultat nous semble étonnant considérant que, de 1971 à 2015, la proportion d'élèves n'ayant pas le français comme langue maternelle a plus que triplé, au Québec, et en particulier que la région montréalaise compte, depuis 2011, un plus grand nombre d'enfants de langue maternelle tierce que d'enfants dont le français est la langue maternelle (office québécois de la langue française, 2017). Il nous apparait donc important que cette diversité linguistique soit mieux représentée dans les bibliothèques de classe. D'ailleurs, plusieurs études montrent les bienfaits des approches d'éveil aux langues, notamment par l'exposition à des albums de différentes langues ou bilingues (Ariaz, 2010 ; Naqvi et al., 2012 ; Rodriguez-Valls, 2011).

La très grande majorité des classes ont des albums d'origine variée. Si toutes les classes ont des albums d'origine québécoise, la proportion moyenne par classe est toutefois plutôt faible $(34,8 \%)$, ce qui nous semble regrettable, au vu des recommandations ministérielles. Par ailleurs, si l'on considère que $32,3 \%$ des albums québécois sont publiés par des éditeurs dits "non reconnus ", la proportion d'ouvrages québécois de qualité est encore plus faible. L'album représentant un outil d'acculturation important pour les élèves issus de l'immigration (Janes et Kermani, 2001), il nous semble nécessaire que le nombre de livres illustrant la réalité de la culture québécoise soit disponible en quantité suffisante dans les classes de maternelle. 


\section{La qualité}

Si la quantité de livres semble avoir légèrement augmenté dans les classes de maternelle, au cours des dernières années, la qualité de ces derniers n'est toutefois pas toujours au rendez-vous. En effet, un peu plus du tiers des albums du corpus (36,3\%) sont publiés par des éditeurs dits "non reconnus». Plusieurs de ces albums sont de type commercial (par exemple, des livres de Bob l'éponge ou de Barbie) et ne représentent pas une littérature de qualité qui permettrait aux enfants de milieux défavorisés d'être en contact avec des livres plus riches que ceux qu'ils peuvent retrouver à la maison. Dans ces circonstances, il n'est pas surprenant de constater qu'un très faible pourcentage des livres $(8,2 \%)$ est recommandé par le répertoire Constellations. Dans le contexte où il n'y a pas de liste de livres prescrits, au Québec, et où, contrairement au matériel didactique (ensembles didactiques et ouvrages de référence), aucun organisme n'approuve le contenu des albums qui se retrouvent dans les bibliothèques de classe, cette situation est préoccupante.

Enfin, en ce qui concerne l'âge du corpus, une forte majorité $(62,5 \%)$ des ouvrages répertoriés ont été publiés à partir de l'an 2000. En outre, les albums du corpus sont en bonne part, en parfait état ou un peu abimés. Cette situation plutôt favorable pourrait s'expliquer par les investissements successifs consentis par le gouvernement québécois, depuis une dizaine d'années, dans le cadre, notamment du Plan d'action sur la lecture qui a permis l'achat de livres.

\section{La qualité globale des bibliothèques de classe}

Nos résultats montrent que le niveau de qualité de la majorité des bibliothèques est jugé faible à minimal, et ce autant pour le critère de la quantité, de la variété que de la qualité, ce qui suggère que l'environnement oral et écrit pourrait ne pas être suffisamment riche pour soutenir les enfants de milieux défavorisés dans leur émergence de l'écrit. Le niveau est particulièrement faible en ce qui concerne deux des indicateurs de la qualité des livres (la proportion d'albums publiés par un éditeur dit "reconnu» et celui des albums recommandés par Constellations). Ces résultats vont dans le même sens que ceux obtenus par Dowohwer et ses collaborateurs (1998) et de Guo et ses collaborateurs (2013) et qui avaient montré que les bibliothèques de classe de maternelle étaient de niveau faible à modéré et ne respectaient pas les recommandations en matière d'environnement littéracique de qualité.

\section{Recommandations et limites}

Ces résultats nous amènent à formuler quelques recommandations. En effet, considérant les sommes investies au cours des dernières années par le gouvernement québécois pour l'achat de livres de classe, le faible niveau de qualité du corpus répertorié, le faible pourcentage des livres du corpus figurant dans le répertoire Constellations, ainsi que la faible proportion de livres québécois de qualité, il y aurait lieu de mettre en place des mesures pour s'assurer que ces sommes soient adéquatement dépensés. La formation des enseignants nous semble un incontournable en ce sens. En effet, il faut mieux former les enseignants à la littérature jeunesse afin qu'ils puissent faire les choix les plus judicieux et susceptibles de développer la posture 
de lecteur des enfants et ainsi contribuer à la formation de sujets lecteurs-scripteurs engagés (Frier et Vadcar, 2017). Il faudrait peut-être également explorer la possibilité de fournir des indications plus précises aux enseignants quant aux critères de choix des œuvres, et ce, tant en termes de quantité, de variété que de qualité ou même proposer des listes, comme c'est le cas en France. Investir des sommes dédiées pour l'achat de livres de classe est, certes, un pas dans la bonne direction, mais l'absence d'outils pour accompagner ou guider les enseignants ou de mécanisme pour s'assurer que les choix faits sont propres à améliorer l'environnement littéracique des classes ${ }^{12}$ nous semble un coup d'épée dans l'eau, en particulier en milieu défavorisé.

Finalement, toute recherche comporte un certain nombre de limites, la nôtre ne fait pas exception. Tout d'abord, il faut mentionner que nous n'avons analysé que les albums accessibles aux enfants, le jour de la collecte. Il est possible que les enseignants gardent rangés les albums plus dispendieux ou de meilleure qualité, ce qui a pu avoir un impact sur la qualité du corpus répertorié. Par ailleurs, comme les enseignants étaient informés de la visite des auxiliaires de recherche, il n'est pas non plus exclu qu'ils aient pu, dans un souci de désirabilité sociale, bonifier leur bibliothèque, le jour de la collecte de données. Cependant, si tel fut le cas, cela n'a pu qu'avoir un impact à la hausse sur le niveau de qualité des bibliothèques qui demeure en moyenne, en dépit de cela, de faible à minimal. Un autre élément dont il faut faire mention est que plusieurs enseignants ont déclaré avoir obtenu des budgets spéciaux au cours de l'année (1 600 \$, en moyenne) afin d'acheter des livres. En effet, en 2018-2019, un budget dédié de $600000 \$$ a été accordé aux écoles par le gouvernement québécois pour l'acquisition de livres pour les classes de l'éducation préscolaire. Toutefois, encore une fois, cela n'a pu que contribuer à rehausser le niveau de qualité observé qui demeure, malgré tout, plutôt insuffisant. Finalement, bien que notre étude permette d'offrir un portrait détaillé de la qualité des bibliothèques de classe, nous n'avons pas analysé le contenu des albums (diversité des thèmes, des styles, des niveaux de complexité, etc.). Nous ne savons pas non plus si les albums reflètent la diversité ethnique et culturelle de la société québécoise actuelle et dans quelle mesure ils sont exempts de stéréotypes de genres ou d'autres formes de préjugés.

\section{Conclusion}

61 Les enfants ayant bénéficié d'un environnement riche en écrits et en occasions d'interagir autour des livres avant leur entrée à l'école ont plus de chance de faire une entrée réussie en lecture et en écriture (National Early Literacy Panel, 2008 ; Sénéchal et LeFevre, 2002), et ce, même si la langue parlée à la maison n'est pas le français (Sénéchal et LeFevre, 2014). Il importe donc que l'école reproduise ces conditions en offrant à tous les enfants un accès à des bibliothèques de classe bien garnies, offrant des albums de genres et d'origines variées et de bonne qualité. Malheureusement, nos résultats suggèrent que pour plusieurs enfants fréquentant des classes de maternelle 4 ans à temps plein en milieu défavorisé (TPMD), la bibliothèque de classe ne répond pas à ces critères de qualité.

D'autres recherches seront nécessaires afin de pouvoir comparer les bibliothèques des classes en milieu défavorisé et en milieu mieux nanti. L'étude de Pianta et ses collaborateurs (2002) montrant que les classes situées en milieu défavorisé présentent 
généralement un niveau plus faible de qualité, il serait pertinent, dans une prochaine étude, de documenter la situation au Québec afin de pouvoir y remédier, au besoin.

\section{BIBLIOGRAPHIE}

Ariaz, S.K. (2010). Vocabulary Acquisition of Bilingual Students through the Implementation of Dialogic Shared Storybook Readinf Techniques [Thèse de doctorat, Université du Texas, ÉtatsUnis]. ProQuest Dissertation \& Theses.

Barnett, W. S., Lamy, C. et Jung, K. (2005). The effects of state prekindergarten programs on young children's school readiness in five states. New Brunswick: National Institute for Early Education Research.

Barnett, W. S., Yarosz, D. J., Thomas, J., Jung, K. et Blanco, D. (2007). Two-way and monolingual English immersion in preschool education: An experimental comparison. Early Childhood Research Quarterly, 22, 277-293.

Bonnéry, S. (2014). Les livres et les manières de lire à l'école et dans les familles : Réflexions à l'occasion de la parution de la liste officielle « maternelle ». Le français aujourd'hui, 185, 47-57. Boudreau, M., Hébert, M-H. et Dubois, J. (2019). Le coin lecture sous la loupe : observation et évaluation de son aménagement au préscolaire. Vivre le primaire, 32(2), 8-11.

Centre de services scolaires de Montréal (s. d.). Guide d'élagage. Récupéré de : https:// cybersavoir.csdm.qc.ca/bibliotheques/files/2017/07/Guide-délagage.pdf

Chapman, M., Filipenko, M., McTavish, M. et Shapiro, J. (2007). First graders' preferences for narrative and/ or information books and perceptions of other boys' and girls' book preferences. Canadian Journal of Education, 30, 531-553.

Charron, A., Plante, I. et Tremblay, O. (accepté). Plaisir, intérêt et motivation à lire. Dans Montésinos-Gelet, I., Dupin de Saint-André, M. et Charron, A. (dir.), L'oral, la lecture et l'écriture à l'éducation préscolaire et au $1^{\text {er }}$ cycle du primaire. Montréal : Chenelière.

Colman, P. (2007). A new way to look at literature : A visual model for analyzing fiction and nonfiction texts. Language Arts, 84, 251-268.

Dowhower, S. L et Beagle, K. G. (1998). The print environment in kindergartens: A study of conventional and holistic teachers and their classrooms in three settings. Reading Research and Instruction, 37, 161-190.

Duke, N. K. et Kays, J. (1998). « Can I say ‘once upon a time’? » Kindergarten children developing knowledge of information book language. Early Childhood Research Quarterly, 13, 295-318.

Fortin, M.-F. et Gagnon, J. (2016). Fondements et étapes du processus de recherche. Montréal : Chenelière.

Frier, C. et A. Vadcar (2017). Mais qu'est-ce qui se passe dans le coin lecture ? Repères, 55, 133-157. 
Fractor, J. S., Woodruff, M. C., Martinez, M. G. et Teale, W. H. (1993). Let's not miss opportunities to promote voluntary reading: Classroom libraries in the elementary school. The Reading Teacher, 46, 476-484.

Giasson, J. (2011). La lecture : apprentissage et difficultés. Montréal : Gaétan Morin.

Giasson, J. et Saint-Laurent, L. (1999). Lire en classe : résultats d'une enquête au primaire. Revue Canadienne de l'éducation, 24(2), 197-211.

Gormley, W. T. et Gayer, T. (2005). Promoting school readiness in Oklahoma: An evaluation of Tulsa's Pre-K program. Journal of Human Resources, 40, 533-558.

Guo, Y., Justice, L. M., Kaderavek, J. N. et McGinty, A. (2012). The literacy environment of preschool classrooms: Contributions to children's emergent literacy growth. Journal of Research in Reading, 35, 308-327.

Guo, Y., Sawyer, B. E., Justice, L. M. et Kaderavek, J. N. (2013). Quality of the literacy environment in inclusive early childhood special education classrooms. Journal of Early Intervention, 35(1), 40-60. Janes, H. et Kermani, H. (2001). Caregivers' Story Reading to Young Children in Family Literacy Programs: Pleasure or Punishment? Journal of Adolescent \& Adult Literacy, 44(5), 458-466.

Kraemer, L. McCabe, P. et Sinatra, R. (2012). The effect of read-alouds of expository text on first graders' listening comprehension and book choice. Literacy Research and Instruction, 51, 165-178.

Lipson, M. Y., Mosenthal, J.H., Mekkelson, J et Russ, B. (2004). Building knowledge and fashioning success on school at time. The Reading Teacher, 57(6), 534-545.

Marjanovic Umek, L., Kranjc, S. Fekonja, U. et Bajc, K. (2006). Quality of the preschool and home environment as a context of children's language development. European Early Childhood Education Research Journal, 14(1), 131-147.

Ministère de l'Éducation, de l'Enseignement supérieur et de la Recherche (s.d.) Guide d'acquisition. Québec : gouvernement du Québec. Récupéré de : http://www.education.gouv.qc.ca/ enseignants/dossiers/lecture/bibliotheques-scolaires/ameliorer-ma-bibliotheque/guidedacquisition/

MELS (2006). Le programme de formation de l'école québécoise. Québec : gouvernement du Québec.

Moss, B. Leone, S. et Dilipplo, M.L. (1997). Exploring the literature of fact: Linking reading and writing through information trade books. Language Arts, 74(6), 418-429.

Morrow, L. M. (1989). Designing the classroom to promote literacy development. Dans D. S. Strickland \& L. M. Morrow (dir.), Emergent literacy: Young children learn to read and write (p. 121-134). Newark International Reading Association.

Morrow, L. M., Dougherty, S. M. et Tracey, D. H. (2019). Best practices in early literacy. Dans L. M. Morrow et L. B. Gambrell (dir.), Best practices in literacy instruction (6 éd., p. 75-103). New York : The Guilford Press.

National Early Literacy Panel . (2008). Developing early literacy. Washington : National Institute for Literacy.

Naqvi R., Thorne, K.J., Pfitscher, C.M., Nordstokke, D. W. et McKeough, A. (2012). Reading dual language books : Improvins literacy skills in linguistically diverse classrooms. Journal of Early Childhood Research, 11(1), 3-15. ; 
Neuman, S. B. et Bredekamp, S. (2000). Becoming a Reader: A Developmentally Appropriate. Dans Strickland, D. S. et Morrow, L. M. (dir.). Beginning Reading and Writing (p. 22-34). New York : Teachers College Press et International Reading Association.

Neuman, S. B. et Celano, D. (2001). Access to print in middle- and low-income communities: An ecological study of four neighborhoods. Reading Research Quarterly, 36, 8-26.

Neuman, S. B.et Roskos, K. (2007). Nurturing knowledge: Building a foundation for school success by linking early literacy to math, science, art, and social studies. New York : Scholastic.

Office québécois de la langue française (2017). Langue et éducation au Québec. Récupéré de : https://www.oqlf.gouv.qc.ca/ressources/sociolinguistique/2017/20170331_etude1.pdf

Pianta, R.C., La Paro, K. M., Payne, C., Cox, M. J. et Bradley, R. (2002). The relation of kindergarten classroom environment to teacher, family, and school characteristics and chil outcomes. The Elementary School Journal, 102(3), 225-238.

Rodriguez-Valls, F. (2011). Family treasures : A dual language book project for negotiating language, literacy, culture, and identity. The Canadian Modern Language Review, 67(1), 123-148.

Salinas, D. (2018). Comment l'école compense-t-elle le désavantage socio-économique ? PISA à la loupe, 76. Paris : Éditions OCDE. Récupéré de : https://doi.org/10.1787/170967ba-fr.

Sénéchal, M. et LeFevre, J. A. (2002). Parental involvement in the development of children's reading skill: A five-year longitudinal study. Child Development, 73(2), 445-460.

Sénéchal, M. et LeFevre, J. (2014). Continuity and Change in the Home Literacy Environment as Predictors of Growth in Vocabulary and Reading. Child Development, 85, 1535-1551.

Smith, M., Brady, J. et Anastasopoulos, L. (2008). Early Language and Literacy Classroom Observation Tool, Pre-K. Baltimore: Brookes Publishing.

Sorin, N. (2003). Traces postmodernes dans les mini-romans et les premiers romans. Dans Lepage, F. (dir). La littérature pour la jeunesse. 1970-2000. (p. 45-67). Montréal : Fidès.

Tracey, D. et Morrow, L. M (2015). Best practices in early literacy. Dans Gambrell, L. B. et Morrow, L. M. (dir.) Best practices in literacy instruction (p.86-105). New York : Guilford press.

Turgeon, E. (2013). Développement et mise à l'essai d'un outil pour analyser des albums jeunesse afin d'élaborer un répertoire d'œuvres québécoises propices au travail interprétatif. [Thèse de doctorat. Montréal : université de Montréal, Canada]. Papyrus. https://papyrus.bib.umontreal.ca/xmlui/ handle/1866/9728

Van der Linden, S. (2007). Lire l'album. Le Puy-en-Velay : l'atelier du poisson soluble.

Vukelich, C., Christie, J., \& Enz, B. (2012). Helping young children learn language and literacy: Birth through kindergarten (3rd ed.). Boston, MA : Pearson.

\section{NOTES}

1. C'est-à-dire les acquisitions en lecture et en écriture faites avant l'apprentissage formel de la lecture.

2. Au Québec, l'éducation préscolaire concerne la maternelle 4 ans et 5 ans, qui n'est pas obligatoire. Les autres structures d'accueil des enfants de moins de 5 ans sont offertes en milieu de garde (Centres de la petite enfance, garderies et services de garde en milieu familial).

3. Au Québec, jusqu'en 2020, la maternelle 4 ans à temps plein était offerte uniquement en milieu défavorisé. 
4. Nous faisons référence à la liste de littérature pour la maternelle française «Pour une première culture littéraire ».

5. Aucune université québécoise n'offre actuellement de cours obligatoire sur la littérature jeunesse dans le parcours de formation initiale des enseignants de niveau préscolaire et primaire.

6. Ce projet a reçu le financement de l'Action concertée sur l'apprentissage de la lecture et de l'écriture du Fonds de Recherche du Québec Société et Culture (FRQSC).

7. Nous avons également évalué la qualité des coins-lecture, mais nous ne présentons pas ces résultats dans le cadre de cet article.

8. Le terme album est utilisé ici pour désigner un format de livre où cohabitent des illustrations et du texte (Van der Linden, 2007), ce dernier adoptant différents genres : abécédaire, récit, documentaire, etc. (Sorin, 2003).

9. https://constellations.education.gouv.qc.ca

10. Au Québec, l'indice de milieu socio-économique permet de déterminer le niveau de défavorisation des écoles primaires et secondaires se trouvant en milieu défavorisé (sur une échelle de 1 à 10, 10 étant le niveau de défavorisation le plus élevé).

11. Grolier est, à l'origine, un éditeur américain, dont une division a été basée au Québec (Montréal) et a publié de nombreux ouvrages en français. Il a fermé ses portes en 2009.

12. Ajoutons qu'il importe également de soutenir les enseignants dans la manière dont ils organisent la rencontre avec ces albums.

\section{RÉSUMÉS}

Plusieurs études ont démontré qu'un environnement physique de qualité soutient le développement des habiletés en émergence de l'écrit des jeunes enfants à l'éducation préscolaire, particulièrement chez les enfants issus de milieux défavorisés et ceux n'ayant pas le français comme langue maternelle. Cet article s'inscrit dans une plus vaste étude menée au Québec, dans 30 classes de maternelle 4 ans à temps plein en milieu défavorisé (TPMD). Nous présentons les résultats d'un volet de l'étude qui visait à décrire et à évaluer l'offre de livres de bibliothèques de classe accessibles aux enfants. Dans l'ensemble, il ressort que le niveau de la qualité des bibliothèques en ce qui concerne l'offre de livres se situe entre le niveau faible et minimal, et ce, autant pour le critère de la quantité, que pour celui de la variété et de la qualité.

Multiple studies have found that a quality physical environment supports the development of kindergarten children's emerging writing abilities, this is particularly true for children from disadvantaged areas and those who did not learn French as a first language. This study is part of a larger research project conducted in 30 full-day kindergartens for 4-year-olds in disadvantaged areas in Quebec. We will present the results of the segment of the study dedicated to describing and evaluating the selection of classroom library books accessible to children. Results show that the levels of quality associated with the book selections of most classrooms libraries ranged from low to minimal as it relates to the criteria of quantity, variety and quality. 
INDEX

Mots-clés : mesure de la qualité, bibliothèque de classe, livres, éducation préscolaire, milieux défavorisés

Keywords : Quality measures, classroom library, books, preschool education, disadvantaged areas.

\section{AUTEURS}

ELAINE TURGEON

Université du Québec à Montréal

ANNIE CHARRON

Université du Québec à Montréal

\section{SARAH JANE MCKINLEY}

Université du Québec à Montréal 